\title{
Some Thinking about Micro-blog in the Improvement of the Library Service in Vocational and Technical College
}

\author{
Huang Jinghua, Hu Xuanzi, Cao Wenliang \\ Dongguan Polytechnic, Dongguan, Guangdong Province, China \\ \{89249134,646252900,25392554\}@qq.com
}

\begin{abstract}
This paper has firstly made a deeply research on the source and character of microblog. Then the application situation of microblog in the vocational and technical college library has been analyzed. On this basis, one kind of microblog system is designed and introduced in the paper. In the end, the problem of the microblog in the application for the library service has also been pointed out. Some improvement suggestions have been proposed at the same time, which can supply some useful exploration for the improvement of library service.
\end{abstract}

Index Terms - blog, microblog, library service

\section{Introduction}

In 2000, blog came into China and has made a greatly development. Some professors declared that China's internet belongs to the blog, at the first decade of the 21 st century. However, the behavior of blog was not the so excellent as the same as people expected.

The development of blog became slower in the next several years. Chinese people firstly knew the blog because of the Mu Zimei affair in 2004. In 2005, blog began to be used in the field of library. But in 2009, some famous blogs, such as the Lao Huaiye microblog, the Zhu Mian, Zhaizhu and so on, have announced to close. And the blog has came into a downturn era[1-2].

The micro-blog made a new era of blog. It can be used in the field of library, and has became a new generation of library network information platform. Microblog is one kind of mini blog. It is a platform based the user relationship, and that can be used to share, transit and obtain information. Users in different area can set up their community by the world wide web, wireless appliction protocol or other kinds of client. The information of microblog can be very small, which can only include 140 words. The earliest microblog of the world is the Twitter in America, which is setup by Evan Williams in 2006. Through the platform, users can issue or refresh information by telephone note. Nowadays, more and more famous microblog web sites have appeared in the internet, such as the Digu net, the sina microblog and so on[3].

\section{Application of Microblog for the Service in Vocational and Technical College Library}

Microblog is a new type of net tool. It has several characters, which is shown as follows[4]:

\section{1) Diversify}

Blog is a network communication platform. Everyone, no matter what they do, how old they are, or the sex they belong to, can enroll their personal microblog by internet. On the blog, they can write diary, issue information, communicate with others, share resource, and so on.

\section{2) Freedom}

Everyone only need to enroll an account, and then can has a personal microblog. They can write everything on it, and express their personal opinion. The content in microblog would not limitted by the phrasing, the format or norm. They can only write one sentence or several sentence. They needn't to worry about the content what they write.

3) Interactive

Compared with the traditional email, microblog is more convenient. Through microblog, users can connect to the net address where the information come from, which can supply a very efficient platform for info rmation communication.

\section{4) Instantaneity}

By internet, microblog can share information instantaneously. In the Wen Chuang earthquake, Twitter issued the information firstly, which is faster than Peng Bo news agency. In Iran election's unrest, Twitter beame to the source of the real time news and took the place of the traditional media. In these two examples, we can find the instantaneity merit of microblog. Especially in the emergency accident's report, microblog has its excellent merit that the traditional media didn't have.

\section{5) Convenience}

People can use every terminal to issue information, such as personal telephone, IM software, outside API interface, and so on. They can also upload some pictures, video to their personal microblog.

\section{6) Originality}

The users of microblog is diversified. The operation of microblog is very easy and its Entry barrier is very slow. The users, who even didn't undergo any training about computer network, can operate proficiently. The operation interface is very convient, which can meet the need of users' creation. Individuation and colloquial style of narration are the microblog's main trend, which has inspired many original network writing.

Microblog has some advantage compared with the traditional media. The application of the microblog to library service can reduce much cost. However, there are only a few libraries applying microblog to the service, which is the 
Chongqing university library, the Xiamen university library, and so on. Their appliction scope is only limited to recommendation service, reference service, user education, promotional activities and so on. The degree and scope of application is still needed to be improved[5-7].

\section{Application of Microblog in Vocational and Technical Library}

Many students in vocational and technical college library were born in 90s. They like to pursue new things. They don't like the content on the book, but feel interested on the internet. Many of them spend much time in the net. According to the students' character, libraries of the vocational and technical college can use microblog to make a innovation[8].

1) The microblog can enhance the interactivity between the readers and library.

Nowadays, many libraries still communicate with readers by traditional methods, such as telephone, message board, email and so on, which is very time costing. Microblog can overcome the disadvantage. The missionary in library can obtain readers' suggestion in the first time, and improve the service.

2) Readers can supervise the service of library.

In library everyone can say everything by microblog. They can express their feeling freely. Library can use the microblog to supervise its service and find the disadvantage timely.

3) microblog can issue information to readers timely.

Microblog has the advantage of timeliness and sharing. Library can issue the information to readers, such as the newest booklist catalogue, the recommend booklist, the note and so on. So the readers can know about the library's recent news.

4)Readers can obtain more knowledge by microblog.

Nowadays, most of the students in vocational and technical college spend much time on internet. According to this character, library can issue the knowledge with some multimedia tools, such as pictures, music, cartoon, and so on. And the students can obtain the knowledge in a loosing environment.

5) Microblog can enhance the communication between readers.

Though microblog's thematic groups, the same questions of different readers can be gathered together, which is convenient for the library's manager to answer the question. It also supplied a platform to the readers who have the same questions. They can communicate with each other and discuss the questions. Then new knowledge can be produced.

\section{Design of Microblog Platform System for Library}

The designed system is realized by Java language, which is based on the $\mathrm{B} / \mathrm{S}$ architecture. The Hibernate and Spring are used to realize the underlying and transaction management. The Ajax is used to be the core technology.

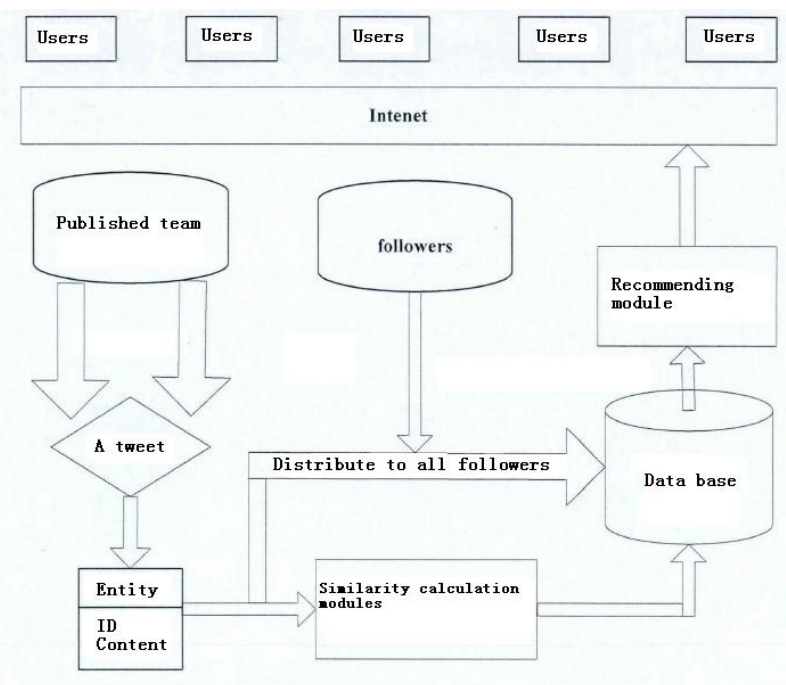

Fig.1 Information release and handling process

The system can be used to the users in Campus Network. The information's release and handling process is shown in fig.1. The whole system includes the similarity calculation module, the recommending module, the system manage module, the microblog manage module and so on, which is shown in fig.2.

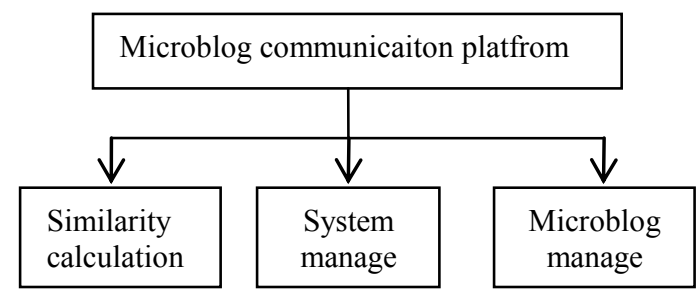

Fig. 2 Microblog commication platform modules

\section{1) Similarity calculation module}

This module is one of the core modules in the system. The content correlation and the user correlation are calculated by Pearson's correlation coefficient. Then the sentence similarity and content correlation is calculated by the weight $1: 1$, and the similarity between microblogs can be obtained.

2) Recommending module

It is also a core module, which includes the recommending based users and project. In order to increase the diversity and accuracy of recommending, the system united the two module to one.

3) System manage module

The module includes module mange, data base maintain, topic maintain, and so on, which can guarantee for applied system failure recovery

\section{4) Micro log manage module}

This module applied a management to user's micro log, pictures, private message, friend list, and so on and that make 
the communication more fluency.

In the aspect of data interaction, the Java Script Object Notation format is taken in the system. It is a data exchange format which is independent of language. All these characters make it to be an ideal data exchange language. It is easy to be read and programmed. Compared with XML, its parse and transmission performance are more wonderful.

The system has taken the MVC architecture, which can make the development and maintain more easily. The Hibernate is used in models to make the data base more persistent. In the aspect of transaction management, Spring is taken. Struts is applied to separate the display layer from control layer, and JSON is taken to be data exchange format between the display layer and control layer. The whole architecture of the system is shown in fig.3.

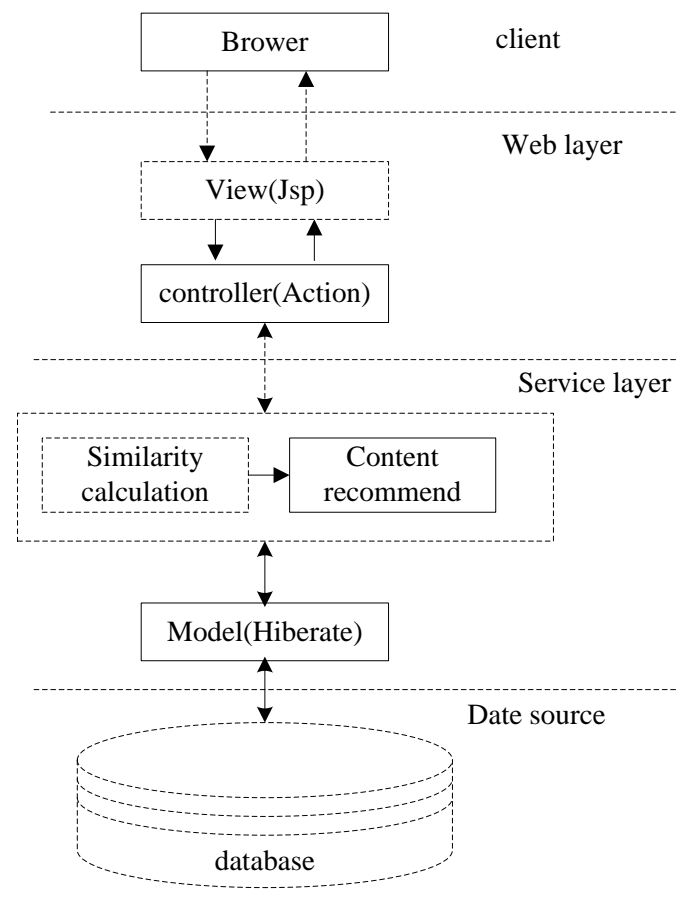

Fig.3 Architecture of the syetem

The data source in the system can be devided into two parts. One is used to store the logic data related with the Sql Server 2008 data base system business, and the other is used to store the file lexicon. Hibernate is applied to map the business logic data, which includes microblog data, user date, microblog similarity data, and so on. The file content will be read into RAM when the system starts, which can greatly improve the system's whole performance.

The service layer and Web layer can be compiled and executed by application server. The service layer is used to manage the system's business logic data, such as the similarity calculation, data conversion and so on. The Spring is applied to manage business in this layer, and the code must follow the MVC design principle. The Web layer is used to separate the display layer from control layer, so the request of client can be responded.
In client layer, users only need to send request to servers by common browser such as the IE. Then they can easily access and operate the system.

\section{Some Problems about the Microblog in Library Service}

Microblog is a new net tool, which appeared in recent year. It has been widely used in the library, and made a great contribution to improve the service quality. However, in the process of actual application, there still exists some problems $[4,8]$.

1) The message doesn't refresh timely.

Nowadays, most of the libraries don't employ the full time staff to maintain the micro-blog. The message is still too little. The refresh speed of information is too slow. So it is very difficult to attract student reader.

2) The content on Micro-blog is still too dull.

Most micro-blog in inland college libraries only contain some words, which didn't integrated some pictures, video, animation. So it is very difficult to attract young man.

3) There exists too much useless information on microblog.

Nowadays, the message on the micro-blog is too much. Much of it is useless. Some of it even jeopardizes national security. It is very difficult for the readers to find the message. Therefore, the managers of library should make more real time supervise to micro-blog.

\section{Some Suggestions about Micro-Blog Applied in Library Service}

Micro-blog is the trend of library service in future, which can greatly improve its service quality. To the application status, some improvement should be done[9-10].

1) The micro-blog platform should be carefully chosen.

Nowadays, there are many micro-blog platform suppliers. Some of them aren't compatible with each other. So the manage should carefully choose the platform, and some important factor should be considered about, such as the compatibility, the safety, and so on.

2) Managers should make more administration to microblog.

The micro-blog has the character of openness. So it is very important for managers to make much more supervisal to the content of micro-blog. Library should set up the special manage method, assign a man to take charge, set norm to the content and supply a better service to readers.

3) Information on micro-blog should refresh timely.

The manage department in library should appoint one man to take charge of the micro-blog's maintain. The man should supervise the content to make sure that the news on the micro-blog is latest and safe. 


\section{Conclusions}

Micro-blog is a new net tool, which appeared in recent years. It has many advantages compared with the traditional media. In this paper, characters of application in vocational and technical college library are firstly discussed. On this basis, one kind of the microblog system is designed and introduced in the paper. However, the application of microplat in every college's library is still on a preliminary stage, and it is inevitable to have some problems. As long as the manage departments in library make more supervise to it, the micro-blog can make a great contribution to the improvement of service quality.

\section{References}

[1] Li Hua, Zhao Wen wei. Micro-blog: the library's next generation network new tool. Books and information, 2009(4):78-82.
[2] Gao Su, Liu Ping. Application of micro-blog in college library. Books and information, 2012(5):106-108.

[3] Zhang Wenyan, Wang Qingrun. Application of micro-blog in library's extension service. Nei Mengu Science and economics, 2011(18):138140.

[4] Li Jian. Application of micro-blog in information service of college library. College library work, 2011(4):44-45

[5] Liu Jianfeng, Liu Chenguang. Discussion about the application of micro-blog in library. Cooperative economics and technology, 2012(449):86-87.

[6] Guan Qin. Some imagine about micro-blog's information dissemination in library. Current information, 2012, 32(2):54-46.

[7] Zhang Nai. Investigate of the application situation of micro-blog in inland library and its development strategy. Information datum job, 2012(1):102-104

[8] Li Yuan, et al. Research on application of Micro-blog in college library. Science information development and technology, 2011(21):35-37.

[9] Guo Feng. Discussion of application of micro-blog in college library and its inspiration. Science information, 2012(29): 267-268.

[10] Fan Bing. Discussion of application of micro-blog in college library. Science information development and technology, 2011(33):13-15. 